\title{
Review of Reactive Power Planning: Objectives, Constraints, and Algorithms
}

\author{
Wenjuan Zhang, Student Member, IEEE, Fangxing Li, Senior Member, IEEE, and \\ Leon M. Tolbert, Senior Member, IEEE
}

\begin{abstract}
The key of reactive power planning (RPP), or Var planning, is the optimal allocation of reactive power sources considering location and size. Traditionally, the locations for placing new Var sources were either simply estimated or directly assumed. Recent research works have presented some rigorous optimization-based methods in RPP. This paper will first review various objectives of RPP. The objectives may consider many cost functions such as variable Var cost, fixed Var cost, real power losses, and fuel cost. Also considered may be the deviation of a given voltage schedule, voltage stability margin, or even a combination of different objectives as a multi-objective model. Secondly, different constraints in RPP are discussed. These different constraints are the key of various optimization models, identified as optimal power flow (OPF) model, security-constrained OPF (SCOPF) model, and SCOPF with voltage-stability consideration. Thirdly, the optimization-based models will be categorized as conventional algorithms, intelligent searches, and fuzzy set applications. The conventional algorithms include linear programming, nonlinear programming, mixed-integer nonlinear programming, etc. The intelligent searches include simulated annealing, evolutionary algorithms, and tabu search. The fuzzy set applications in RPP address the uncertainties in objectives and constraints. Finally, this paper will conclude the discussion with a summary matrix for different objectives, models, and algorithms.
\end{abstract}

Index Terms-Evolutionary algorithms (EAs), fuzzy sets, linear programming (LP), mixed integer nonlinear programming (MINLP), multi-objective optimization, nonlinear programming (NLP), OPF, reactive power optimal allocation, reactive power planning, simulated annealing (SA), security-constrained OPF (SCOPF), security constrained OPF with voltage-stability consideration (SCOPF-VS), tabu search (TS), voltage stability margin.

\section{INTRODUCTION}

O PTIMAL allocation of Var sources, such as capacitor banks, Static Var Compensators (SVC), and STATic COMpensators (STATCOM), is a critical component in reactive power planning (RPP) or Var planning. Traditionally, the locations for placing new Var sources were either simply estimated or directly assumed. Recent research has presented some rigorous optimization-based methods to address RPP. Due to the complicated objective functions, constraints, and solution algorithms, RPP is identified as one of the most challenging problems in power systems.

Manuscript received January 29, 2007; revised June 21, 2007. This work was supported by Oak Ridge National Laboratory. Paper no. TPWRS-00044-2007. W. Zhang is with California ISO, Folsom, CA 95763 USA.

F. Li and L. M. Tolbert are with the Department of Electrical Engineering and Computer Science, The University of Tennessee, Knoxville, TN 37996 USA (e-mail: fli6@utk.edu)

Digital Object Identifier 10.1109/TPWRS.2007.907452
The following assumptions are considered while formulating the Var planning problem in the literature.

- The system is balanced.

- The active and reactive power represent fundamental frequency powers, and additional powers at harmonic frequencies are negligible.

- The size of the Var source is treated as a continuous variable; however, it is in fact discrete.

- The reactive capability of a generator is portrayed by the conventional PQ-diagram, but for the planning study, it is usually sufficient to assume a fixed upper limit $Q_{\max }$ relevant to the generator MW output.

After a detailed study of the large amount of previous works, we believe that an informative and succinct literature review in RPP should summarize the objective function model, the constraint model, and the mathematical algorithms. These three components are briefly discussed next.

The objective function of RPP may be cost-based, which means to minimize the possible cost associated with RPP such as variable and fixed Var installation cost, real power loss cost, and/or fuel cost. Other possible objectives may be to minimize the deviation from a given schedule of a control variable (such as voltage) or to maximize voltage stability margin. It is also reasonable to use a multi-objective (MO) model as the goal of the RPP formulation.

The constraints in RPP are even much more complicated than the objective functions. Conventional constraints may include the normal state (base case) power-flow limits and the contingency state power flow limits. However, more recent works proposed to include the voltage stability limits, under both normal state and contingency state, due to the increased pressure of voltage stability and stressed transmission systems. These different constraints are the key of the classification of various optimization models, identified as optimal power flow (OPF) model, security-constrained OPF (SCOPF) model, and SCOPF with voltage stability constraints (SCOPF-VS), the present state-ofthe-art in RPP. In some works, the consideration of contingency analysis and voltage stability may be included in the objective functions. The relationship in terms of feasible region among the three formulations is shown in Fig. 1. It should be noted that in both theory and practice, one of the objective models may be combined with one of the constraint models to formulate the RPP problem. However, the constraint model is the key to the classification of the three optimal power-flow models.

The mathematical solution of RPP is also very challenging due to a large number of variables and uncertain parameters. Based on the objective and constraint formulation, RPP could be mathematically formulated with variables and equations that are 


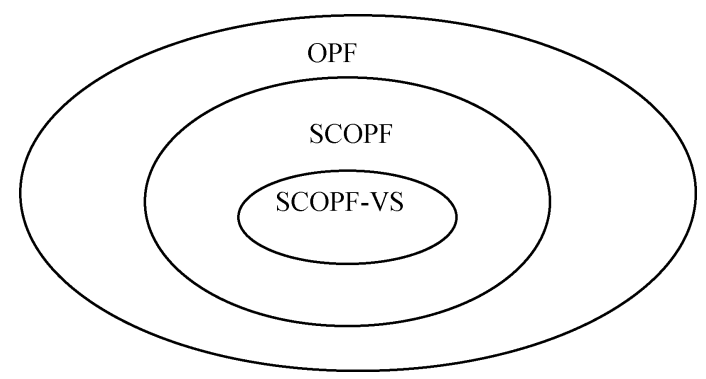

Fig. 1. Relationship of different RPP models.

partially discrete, partially continuous, nondifferentiable, and nonlinear. There are no known ways to find the exact global solution for this complicated optimization problem in a reasonable time. The solution techniques for RPP have evolved over many years, and dozens of approaches have been developed, each with its particular mathematical and computational characteristics [1]. The majority of the techniques discussed in the literature of the last 20 years may be classified as one of three categories: conventional methods, intelligent searches, and fuzzy set applications. The conventional methods include linear programming (LP), nonlinear programming (NLP), mixed-integer nonlinear programming (MINLP), etc. The intelligent-search-based methods, such as simulated annealing (SA), evolutionary algorithms (EAs), and tabu search (TS), have received widespread attention as possible techniques to obtain the global optimum for the RPP problem. However, these heuristic methods need more computing time in general. Also, fuzzy set theory has been applied to RPP to address uncertainties in objectives and constraints.

This paper is organized as follows. Section II illustrates the possible objectives in the RPP literature. Section III presents the different constraint models such as OPF, SCOPF, and SCOPF-VS. Section IV presents the mathematic algorithms to solve the above models. Section $\mathrm{V}$ presents the conclusion with a matrix to match the objectives, the constraint models, and the solution algorithms to different papers.

\section{ObJectives of Reactive Power PlanNing}

The majority of the RPP objectives were to provide the least cost of new reactive power supplies. Many variants of this objective include the cost of real power losses or the fuel cost. In addition, some technical indices such as deviation from a given voltage schedule or the security margin may be used as objectives for optimization. The detailed discussions are presented as follows.

\section{A. Minimize Var Cost}

Generally, there are two Var source cost models for minimization. The first formulation is to model Var source costs with $C_{1} \cdot Q_{c}$ that represents a linear function with no fixed cost [2]. Apparently, this model considers only the variable cost relevant to the rating of the newly installed Var source $Q_{c}$ and ignores the fixed installation cost. The common unit for $C_{1}$ is $\$ /$ (MVar - hour). This means the costs of two 200-MVar Var sources are exactly the same as one 400-MVar source. This formulation would always bias a solution toward placement of several smaller sizes sources instead of a small number of larger ones.

A better formulation with the format $\left(C_{0}+C_{1} \cdot Q_{c}\right) \cdot x$ [3]-[5], [35]-[38] is to consider the fixed cost, $C_{0}$ (\$/hour), which is the lifetime fixed cost prorated to per hour, in addition to the incremental/variable cost, $C_{1}(\$ / \mathrm{MVar} \cdot$ hour $)$. This is a more realistic model of Var cost, but this would complicate the problem from a nonlinear programming (NLP) to a mixed-integer NLP (MINLP), because there is a binary variable $x$ to indicate whether the Var source will be actually installed or not. The slight difference in the cost model, however, leads to dramatic difference in the optimization model and the corresponding mathematics technique to solve it.

As a result, the RPP model with the first Var cost function as an objective is a traditional LP or NLP problem. However, the second one is a MINLP problem, and some special techniques are needed for it. More details will be found in Section IV about the algorithms.

\section{B. Minimize Var Cost and Real Power Losses}

This is also a common RPP objective. Based on the Var cost model presented in the previous subsection, the objective under this category may be divided into two groups: to minimize $C_{1}\left(Q_{c}\right)+C_{2}\left(P_{\text {loss }}\right)$ [6], [7], [28], [31], [32],[51] and to minimize $\left(C_{0}+C_{1} Q_{c}\right) \cdot x+C_{2}\left(P_{\text {loss }}\right)$ [9]-[13], [30], [50]. Here $C_{2}\left(P_{\text {loss }}\right)$ represents the cost of real power loss.

Reference [31] considers the real power losses consumed not only in the base case but in all contingency cases. So the objective can be written as follows:

$$
\min F=C_{1}\left(Q_{c}\right)+\sum_{k=0}^{N_{C}} C_{2}\left(P_{\text {loss }}\right)_{k}
$$

where $k\left(=0,1, \cdots, L, \ldots N_{c}\right)$ represents the $k^{\text {th }}$ operating case. Here, considered are the base case $(k=0)$, the contingency cases under preventive mode $(k=1, \cdots L)$, and the contingency cases under corrective mode $\left(k=L+1, \cdots N_{c}\right)$.

\section{Minimize Var Cost and Generator Fuel Cost}

As an alternative to the cost of real power loss, the fuel cost is adopted as a direct measure of the operation cost. The minimization of real power loss cannot guarantee the minimization of the total fuel cost in general. Instead, minimization of the total fuel cost already includes the cost reduction due to the minimization of real power loss.

This objective consists of the sum of the costs of the individual generating units

$$
C_{T}=\sum_{i=1}^{n} f_{i}\left(P_{g i}\right)
$$

where $f_{i}\left(P_{g i}\right)=a_{0 i}+a_{1 i} P_{g i}+a_{2 i} P_{g i}^{2}$ is the common generator cost-versus-MW curves approximately modeled as a quadratic function, and $a_{0 i}, a_{1 i}, a_{2 i}$ are cost coefficients.

The objective $C_{1}\left(Q_{c}\right)+C_{T}$ is used in [14], [15]. A MINLP optimization model with objective $\left(C_{0}+C_{1} Q_{c}\right) \cdot x+C_{T}$ is used in [16] and [33]. 


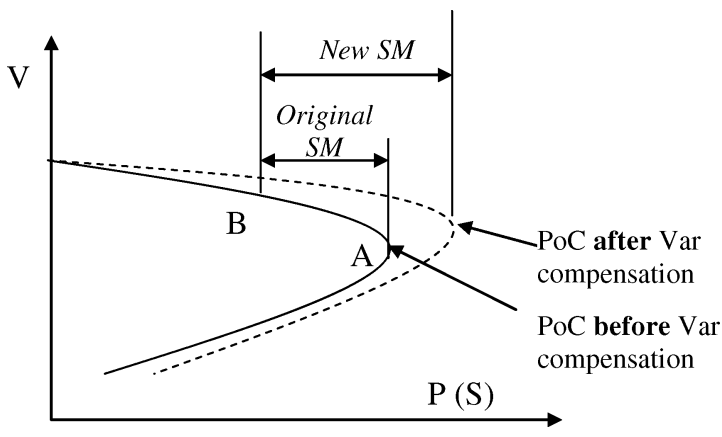

Fig. 2. Voltage stability curve.

\section{Minimum Deviation From a Specified Point}

Different from the previous three cost-oriented objective functions, this objective is usually defined as the weighted sum of the deviations of the control variables, such as bus voltages, from their given target values. The target values correspond to the initial or specified operating points. Minimizing voltage deviation, i.e., $\sum_{i}\left(V_{\text {imax }}-V_{i}\right)$, where the subscript $i$ represents different buses for voltage regulation, is modeled as the objective function in [17].

\section{E. Voltage Stability Related Objectives}

Traditionally in RPP, the feasible operation has been translated as observing voltage profile criteria to ensure that the system voltage profile is acceptable for system normal and post-contingency conditions. However, when many power systems are more stressed, voltage is a poor indicator of proximity to system collapse conditions. Thus, the incorporation of voltage stability in RPP has become essential. Several previous works considered voltage stability related measures as objectives in RPP.

The main function of shunt reactive power compensation is to provide voltage support to avoid voltage instability or a largescale voltage collapse. As shown in Fig. 2, voltage stability is usually represented by a P-V (or S-V) curve. The nose point of the P-V curve is called the point of collapse $(\mathrm{PoC})$, where the voltage drops rapidly with an increase of load. PoC is also known as the equilibrium point, where the corresponding Jacobian becomes singular. Hence, power-flow solution fails to converge beyond this limit, which indicates voltage instability and can be associated with a saddle-node bifurcation point.

These instabilities are usually local area voltage problems due to the lack of reactive power. Therefore, one objective can be to increase the static voltage stability margin (SM) defined as the distance between the saddle-node-bifurcation point and the base case operating point. SM can be expressed as follows:

$$
S M=\frac{\sum_{i} S_{i}^{\text {critical }}-\sum_{i} S_{i}^{\text {normal }}}{\sum_{i} S_{i}^{\text {critical }}}
$$

where $S_{i}^{\text {normal }}$ and $S_{i}^{\text {critical }}$ are the MVA loads of load bus $i$ at normal operating state B and the voltage collapse critical state (PoC) A as shown in Fig. 2, respectively. One could expect an improvement in the stability of the system for that operating point.
The objective in [18] is to maximize the minimum singular value of the power flow Jacobian matrix, which is used as a static voltage collapse index. In [19], the Var planning problem is formulated as maximization of the active power voltage stability margin (P-margin) in order to improve the static voltage stability.

\section{F. Multi-Objective (MO)}

Several single objectives have been discussed above, even though some are essentially weighted sums of different objectives such as active power losses and Var cost. Usually these weights are easily decided by translating the real power losses into \$. However, the goal of RPP is to provide the system with efficient Var compensation to enable the system to be operated under a correct balance between security and economic concerns. If both security and cost are included in the same objective function, then the weights can not be decided directly and easily. The objective in [20], [21] includes Var investment cost minimization, power loss reduction, and voltage deviation reduction as follows:

$\operatorname{Min} F=\left(C_{0}+C_{1} Q_{c}\right) \cdot x+C_{2}\left(P_{\text {loss }}\right)$ and $\operatorname{Max}\left|V_{\text {imax }}-V_{i}\right|$

In [22], the MO model is given as follows:

$$
\begin{aligned}
\operatorname{Min} F= & 10 \sum(\text { voltage violation in p.u. })^{2} \\
& +5 \sum(\text { generator Var violation in p.u. })^{2} \\
& + \text { power losses in p.u. }
\end{aligned}
$$

Another MO RPP model was proposed in [34], in which the objective is designed as minimizing weighted sum of Var cost, real power losses, and deviation of voltage + deviation of line flow as follows:

$$
\begin{aligned}
\text { Min } F & =\left(C_{0}+C_{1} Q_{c}\right) \cdot x+C_{2}\left(P_{\text {loss }}\right) \\
& +\rho_{1} \sum_{i}\left(\frac{\left|V_{i}-V_{\text {ispec }}\right|}{V_{i \max }}\right)^{2}+\rho_{2} \sum_{l}\left(\frac{\left|S_{l}-S_{l s p e c}\right|}{S_{l \max }}\right)^{2}
\end{aligned}
$$

where $V_{i}$ is the voltage magnitude in bus $i, V_{\text {ispec }}$ is the specified voltage magnitude in bus $i$, and $V_{\text {imax }}$ is the maximum allowable voltage deviation limit at bus $i . S_{l}$ is the MVA flow through line $l, S_{l s p e c}$ is the MVA capacity limit of line $l$ and $S_{\text {lmax }}$ is a specified allowable line flow deviation limit. $\rho_{1}$ and $\rho_{2}$ are weights for different objectives.

Chen and Liu [23]-[26] incorporated the voltage stability margins (S-margin) directly into the objective function and created a comprehensive MO model as follows:

$$
\begin{aligned}
& \min F_{1}=\left(C_{0}+C_{1} Q_{c}\right) \cdot x+C_{2}\left(P_{\text {loss }}\right) \\
& \min F_{2}=1-S M \\
& \min F_{3}=\sum_{i} \frac{\Phi\left(\left|V_{i}-V_{i}^{\text {ideal }}\right|-\Delta v_{i}\right)}{V_{i}}
\end{aligned}
$$

In the above formulation, $F_{1}$ is to minimize operation cost caused by real power losses and Var source investment cost. $F_{2}$ is to maximize the voltage stability margin $(\mathrm{SM}) . F_{3}$ is to minimize the voltage magnitude deviation, where $\Phi(x)=0$ if $x<0$; and $\Phi(x)=x$ otherwise; $V_{i}^{\text {ideal }}$ is the specifically 
desired voltage at load bus $i$ and is usually set to be 1 p.u.; and $\Delta v_{i}$ is the tolerance of maximum deviation in the voltage.

In addition to the above formulations, [27] presents another example to treat the voltage stability as one objective in the MO problem with the three objectives: $\min F_{1}=C_{1} Q_{c}$, min $F_{2}=$ $C_{2}\left(P_{\text {loss }}\right)$, and $\max F_{3}=$ the maximum loadability associated with the critical state.

\section{Constraints of Reactive Power Planning}

As an optimization problem, RPP considers power flow equations as the key constraints conventionally, while achieving a certain objective function as discussed in the previous section. This model, optimizing an objective function while respecting the power flow as a constraint, is called the OPF model. More advanced research has concluded that not only normal state power flow, but also the contingency power flow, should be considered. This leads to the model of SCOPF. Recently, due to a necessity to consider voltage stability, a few papers incorporated the static voltage stability (VS) margin in the RPP OPF objective. This provides more realistic solutions for RPP, but it cannot guarantee whether the voltage stability margin still exists when contingencies actually happen. Thus, it is preferable to count on the voltage stability constraints in the contingency states. A milestone in RPP, the SCOPF-VS model, was reached in the late 1990's.

As stated in Section I, theoretically and practically, different objective models may be combined with one of the constraint models to formulate the RPP problem except that voltage stability may be modeled either as an objective function or as constraints. However, the constraint model is the key to the classification of the three optimal power flow models. That is why the RPP models are classified mainly based on constraint formulations.

\section{A. OPF Model for RPP}

1) Introduction to Optimal Power Flow: As shown in [1]-[27], RPP is typically identified as an optimization problem in power systems, known as OPF. There are six variables for each bus: generator active and reactive power output, active and reactive load, and bus voltage magnitude and angle. At the same time, there are two equations related to each bus, active power balance and reactive power balance equations. In the conventional power flow solution, four of the six variables are known or specified, the other two are unknown. For a system with $n$ buses, power flow solves the $2 n$ unknowns from the $2 n$ equations. Using a suitable mathematical algorithm, a feasible solution can be obtained.

However, conventional power-flow algorithms do not automatically minimize any objective functions such as fuel cost or real power transmission losses. They only obtain a single feasible solution. Hence, the need for an OPF arose in the early 1960 's to achieve a desired objective, rather than just a feasible solution. Some controllable power system elements of the $4 n$ known variables in power flow are relaxed into a bounded range. As a result, an infinite number of feasible solutions are obtained by solving the $2 n$ equations for more than $2 n$ unknown variables.
All these feasible solutions construct a feasible region. An optimal solution can be selected from the feasible region to obtain a desired objective by adjusting the optimal setting for the controllable variables with respect to various constraints. The OPF is a static constrained nonlinear optimization problem, and its development has closely followed advances in numerical optimization techniques and computational methods.

2) Formulation of $O P F$ : OPF is formulated mathematically as a general constrained optimization problem as

$$
\begin{array}{ll}
\text { Minimize } & f(u, x) \\
\text { Subject to } & g(u, x)=0 \\
& h(u, x) \geq 0
\end{array}
$$

where $u$ is the set of controllable variables in the system; $x$ is the set of dependent variables called state variables; objective function (1) is scalar; equalities in (2) are the conventional power flow equations and occasionally include a few special equality constraints such as the limit of the number of potential Var compensators; and inequalities in (3) represent the physical limits on the control variables $u$, and the operating limits on the power system.

The control variables $u$ may be defined as follows.

- Generator active power output.

- Regulated bus voltage magnitude.

- Variable transformer tap setting.

- Phase shifters.

- Switched shunt reactive devices.

- Load to shed in special conditions.

The state variables $x$ may be defined as follows:

- Voltage magnitudes at load buses.

- Voltage phase angle at every bus.

- Line flows.

3) Details of OPF Constraints: The details of the OPF constraints, represented in (2) and (3) are discussed here. They may be classified as the conventional power flow equality constraints, physical limits of the control variables $u$, physical limits of the state variables $x$; and other limits such as power factor limits.

- Power flow constraints

$$
P_{g i}-P_{l i}-P(V, \theta)=0 \quad \text { (active power balance) }
$$

$Q_{g i}+Q_{c i}-Q_{l i}-Q(V, \theta)=0 \quad$ (reactive power balance).

- Control variables limits

$P_{g i}^{\min } \leq P_{g i} \leq P_{g i}^{\max } \quad$ (active power generation limits)

$V_{g i}^{\min } \leq V_{g i} \leq V_{g i}^{\max } \quad$ (PV bus voltage limits)

$T_{l}^{\min } \leq T_{l} \leq T_{l}^{\max } \quad$ (transformer tap change limits)

$Q_{c i}^{\min } \leq Q_{c i} \leq Q_{c i}^{\max } \quad$ (Var source size limits).

- State variables limits

$Q_{g i}^{\min } \leq Q_{g i} \leq Q_{g i}^{\max } \quad$ (reactive power generation limits)

$V_{i}^{\min } \leq V_{i} \leq V_{i}^{\max } \quad$ (PQ bus voltage limits)

$\left|L F_{l}\right| \leq L F_{l}^{\max } \quad$ (line flow limit).

- Other limits

Power factor constraints; etc. where 
$P_{g i} \quad$ generator active power output;

$P_{l i} \quad$ load active power;

$Q_{g i} \quad$ generator reactive power output;

$Q_{c i} \quad$ Var source installed at bus $i$;

$Q_{l i} \quad$ load reactive power;

$V_{g i} \quad$ PV bus voltage;

$T_{l} \quad$ transmission tap change;

$V_{i} \quad$ bus voltage;

$L F_{l} \quad$ transmission line flow;

\section{B. SCOPF Model for RPP}

Security constrained optimization was introduced in the 1960s and early 1970s. Alsac and Stott in 1974 introduced SCOPF with exact ac power flow equations [39]. More work has been done on SCOPF since then as evidenced in [40]-[43]. Also, a number of previous works applied SCOPF for RPP [28]-[34]. With the development of the SCOPF model, the goal of RPP is extended to determine a minimum cost allocation plan of new Var resources in terms of size and location so as to guarantee feasible operation both under normal conditions and after contingencies.

1) Introduction to Security Constrained OPF (SCOPF): A conventional OPF minimizes an objective function such as Var costs and/or losses, while maintaining the nonlinear power-flow balance and the system variables, such as voltages, tap ratios, active and reactive generations, and line flows within the specified limits under normal state (base case). However, the optimal solution from OPF may lead to a violation of operating limits under some credible contingency cases. Usually contingency analysis is performed in order to respond to each insecure contingency case. With the hope to integrate the security (contingency) constraints into OPF formulation, the SCOPF model was developed as an important step forward in OPF technology.

The SCOPF model extends the capability of the OPF model to include the effect of potential contingencies, such as outages of transmission branches, generation units, or substation buses. The contingencies contribute additional constraints to the base case OPF problem in that: 1) the nonlinear power flow equations must be observed under contingency cases and 2) all control variables must be within prescribed emergency limits. Therefore, SCOPF minimizes the base case or pre-contingency objective function while observing both the pre- and post-contingency constraints. In other words, SCOPF determines an optimal operating point, such that in the event of any contingency of a given list, the post-contingency states will remain secure (within operating limits).

2) Formulation of SCOPF: SCOPF formulation is as follows: the engine of SCOPF is the basic OPF problem. The original OPF formulation in (1), (2), and (3) can be expanded to include contingency constraints, thus we have

$$
\begin{array}{ll}
\text { Minimize } & f\left(u^{0}, x^{0}\right) \\
\text { Subject to } & g^{k}\left(u^{k}, x^{k}\right)=0, \text { for } k=0,1, \ldots, N_{c} \\
\text { and } & h^{k}\left(u^{k}, x^{k}\right) \geq 0, \text { for } k=0,1, \ldots, N_{c}
\end{array}
$$

where superscript " 0 " represents the pre-contingency case (base case) being optimized, and superscript " $k$ " $(k>0)$ represents the post-contingency states for the $N_{c}$ contingency cases.

Note that the SCOPF formulation considers both the pre- and post-contingency power flows and all constraints in those states should always be satisfied. The post-contingency constraints are of the same dimensional order as those of the pre-contingency case. If there are $m$ total constraints in a given base case OPF, there will be $(k+1) \bullet m$ constraints in a SCOPF formulation with $k$ contingencies. As a result, a 1000-bus system with nine contingencies in SCOPF would be equivalent to a 10000-bus OPF model. It is apparent that the complexity of SCOPF increases greatly compared with the OPF problem.

3) Security Level and Operation Modes: The SCOPF is aimed at scheduling the power system controls to achieve operation at a desired security level, while optimizing an objective function such as cost. SCOPF usually treats security level 1-“secure" and level 2-“correctively secure", which are in secure state. Level-1 security is the ideal operating requirement but is more expensive than operating with a level-2 security.

Under the security level 1 corresponding to the preventive mode, all loads should be supplied and no operating limits should be violated in the event of a contingency. In other words, level 1 has the ideal security, and a power system survives any of the relevant contingencies without relying on any post-contingency corrective action.

Under the security level 2 corresponding to the corrective mode, any violations caused by a contingency can be corrected by appropriate control action without loss of load, within a specified period of time [42].

Note that the contingency constraints model in SCOPF represents steady-state contingencies. So, the solution of SCOPF is not assured to be transiently stable.

\section{SCOPF Considering Voltage Stability (SCOPF-VS)}

As mentioned in Section II-E, there are growing interests to include voltage stability as constraints in RPP, since reactive power compensation can increase voltage stability margin. Different from Sections II-E and II-F, which considers voltage stability in RPP objective functions, this section will discuss the research considering voltage stability as constraints in RPP.

1) Voltage Stability Analysis (VSA) of Power Systems: Before the discussion of voltage stability as constraints, it should be helpful to review some analytical methodologies that have been proposed and are currently used for voltage stability analysis such as P-V and V-Q curve analysis, determination of how far the system is operating from the PoC using continuation power flow (CPF) [44] based on bifurcation theory [45], or OPF approach considering a given pattern of load increase and generator sharing [35]-[38]. It is demonstrated in [46] that bifurcation theory is basically equivalent to some typical optimization-based methodologies. The computation of PoC can be formulated as an optimization problem, known as total transfer capability (TTC).

Several computational methods based on bifurcation theory have been shown to be efficient tools for VSA. However, it is technically difficult to introduce operational limits and computationally expensive to use the continuation method, especially 


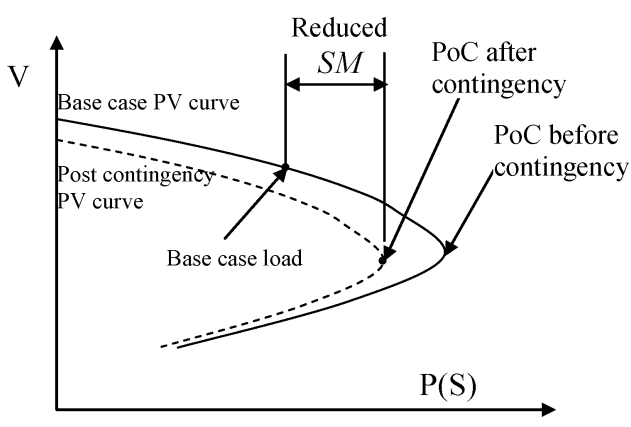

Fig. 3. PV curve for base case and contingency.

for large systems with multiple limits. Using optimization techniques for these types of studies present several advantages, especially due to their capability to handle constraints.

2) Indirect Use of Voltage Stability Margin: Voltage stability margin may be converted into several voltage stability indices, which can be used to select weak nodes as new Var source locations or as candidate locations for the follow-up OPF process [18], [47]. Ajjarapu, et al. introduce a voltage stability index [47] from CPF method to serve as an indirect measure to indicate the closeness to $\mathrm{PoC}$ from the present system load level, which is defined as the ratio of variation of load parameter from $\mathrm{CPF}$ and the voltage variation. The voltage stability index is used to choose the weak buses in order to reduce the complexity by reducing the candidate locations. Reference [17] uses sensitivity method and voltage stability margin index to rank the locations of the new Var source, but the result may be different. Then, parallel analytical criteria are employed in an analytic hierarchical process (AHP) to accommodate the ranks.

In general, these approaches can be viewed as a suboptimal solution for computational efficiency because it excludes some scenarios through some indices related to voltage stability.

3) SCOPF-VS: Conventionally, SCOPF does not consider the change of $\mathrm{PoC}$ after possible compensators are connected. Thus, it is preferable to include the voltage stability constraints in the contingency states. This model is referred to as SCOPF-VS.

References [35]-[38] incorporate a security margin (SM) as the constraint for two reasons. First, this is to guarantee the voltages at all buses lie within a predefined range at normal condition. Secondly, this is to ensure the operating point even after contingency should be away from $\mathrm{PoC}$ at least by a pre-defined "distance" measured in MVA, which is also called S-margin/ MVA-margin. Fig. 3 shows the PV curves before and after contingency and the reduced SM at post-contingency.

It is not necessary to use a separate tool to calculate PoC. Instead, two sets of constraints, one for normal operating state and the other for critical state can be used in this model such that the security margin of post-contingency satisfies the preset secure distance.

The complexity of the voltage stability constrained model lies in the requirement of having two sets of network variables and power flow constraints corresponding to the "normal operation point" and "PoC". Reference [38] explores the use of statistical linear/quadratic approximation function of the path of $\mathrm{PoC}$ as shown in Fig. 4 as an alternative of the variables and constraints

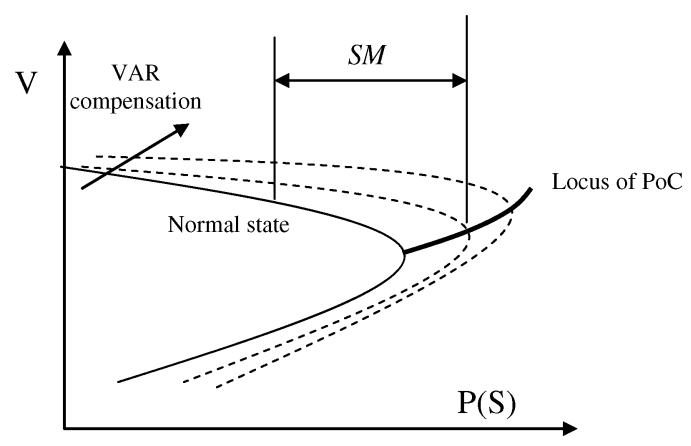

Fig. 4. Locus of $\mathrm{PoC}$ with reactive compensation.

for PoC state. However, if we increase the number of candidate buses, the procedure will involve evaluating a high number of Var support configurations to capture the interaction of Var compensators at different locations.

\section{Mathematical Algorithms for Reactive POWER PLANNING}

In the previous two sections, the objective functions and the constraints are discussed as the optimization formulation of RPP. This section will discuss the mathematical algorithms to solve the optimization-based RPP problem. The algorithms may be classified into three groups: conventional optimization methods including nonlinear programming (NLP), linear programming (LP), and mixed integer programming (MIP); intelligence searches such as simulated annealing (SA), evolutionary algorithms (EAs), and and tabu search (TS); and fuzzy set applications to address uncertainties in objectives and constraints.

It should be noted that there are a number of optimization solvers available such as MINLP solver DICOPT++ and NLP solver MINOS. There are also high-level programming language tools available such as GAMS, an optimization modeling language that may be linked with many different solvers.

\section{A. Conventional Methods}

1) Linear Programming (LP): The LP-based technique is used in [4], [5], [8], [11], [14], and [29] to linearize the nonlinear reactive power optimization problem. The LP approach has several advantages. Firstly, it is reliable, especially regarding the convergence properties. Secondly, it can quickly identify infeasibility. Thirdly, it accommodates a large variety of power system operating limits, including the very important contingency constraints. Nevertheless, despite a number of advantages, its range of application in the OPF field has remained somewhat restricted because of the inaccurate evaluation of system losses and insufficient ability to find an exact solution as opposed to an accurate nonlinear power system model.

2) Nonlinear Programming (NLP): To solve a nonlinear programming problem, the first step in this method is to choose a search direction in the iterative procedure, which is determined by the first partial derivatives of the equations (the reduced gradient). Therefore, these methods are referred to as the first-order methods such as the generalized reduced gradient (GRG) method [54]. 
The successive quadratic programming (SQP) [19] and Newton's method [48] require the computation of the second-order partial derivatives of the power-flow equations and other constraints (the Hessian) and are therefore called second-order methods.

When applied to large-scale power systems, these NLP implementations characteristically suffer from the following two major problems.

- Even though it has global convergence, which means the convergence can be guaranteed independent of the starting point, a slow convergent rate may occur because of zigzagging in the search direction.

- Different "optimal" solutions are obtained depending on the starting point of the solution because the method can only find a local optimal solution.

3) Mixed-Integer Nonlinear Programming (MINLP): Theoretically RPP can be formulated as a MINLP optimization method with integer variables with values of 0 or 1 to represent whether a new Var source should be installed. Reference [3] presents the recursive mixed-integer programming technique using an approximation method. Reference [4] employs branch and bound $(\mathrm{B} \& \mathrm{~B})$ method, which is a typical method for integer programming. A decomposition technique is then employed in [4] to decompose the problem into a continuous problem and an integer problem.

Decomposition methods can greatly improve the efficiency in solving a large-scale network by reducing the dimensions of the individual subproblems. The results show a significant reduction of the number of iterations, required computation time, and memory space. Also, decomposition allows the application of a separate method for the solution of each subproblem, which makes the approach very attractive. Benders decomposition method (BDM) is applied in [31] and [33].

\section{B. Intelligent Searches}

Recently, heuristic methods based on intelligent search have been used in RPP to deal with local minimum problems and uncertainties. Increasingly, these heuristic methods are being combined with conventional optimization methods to solve the RPP problem.

1) Simulated Annealing (SA): From a mathematical standpoint, SA, as introduced by Kirkpatrick, Gelatt, and Vecchi, is a stochastic algorithm aimed at minimizing numerical functions of a large number of variables. It allows random upward jumps at judicious rates to provide possible escapes from local energy wells [55]. Therefore, it converges asymptotically to the global optimal solution with probability one.

The two-layer simulated annealing (TLSA) [20], and the hybrid expert system simulated annealing (ESSA) [21] are proposed to improve the CPU time of SA while retaining the main characteristics of SA. SA is combined with many other approaches such as the genetic algorithm (GA) [12], the $\varepsilon$-constraint method [26], [34], the goal-attainment approach [23], the weighted-norm approach [24], and the fuzzy logic in [25] to deal with MO problems.

2) Evolutionary Algorithms (EAs): Natural evolution is a population-based optimization process. An EA is different from conventional optimization methods, and it does not need to differentiate cost function and constraints. Theoretically, like Simulated Annealing, EAs converge to the global optimum solution with probability one. EAs, including evolutionary programming (EP), evolutionary strategy (ES), and GA are artificial intelligence methods for optimization based on the mechanics of natural selection, such as mutation, recombination, reproduction, crossover, selection, etc. Reference [16] compares the three algorithms, EP, ES, and GA for the same RPP model.

GA is used to select the location and the rating of the Var source and SLP is used to solve operational optimization subproblems as a hybrid method in [5], [11], and [14]. GA is used in [22] to handle the MO model.

3) Tabu Search (TS): The TS algorithm was first developed independently by Glover and Hansen both in 1986 for solving combinatorial optimization problems [56]. It is an iterative search algorithm, characterized by the use of a flexible memory. It is able to eliminate local minima and to search areas beyond a local minimum. TS is proposed in [7], [13] for the optimal RPP problem.

\section{Application of Fuzzy Set Theory}

The data and parameters used in RPP are usually derived from many sources with a wide variance in their accuracy. For example, although average load is typically applied in RPP, the actual load should follow some uncertain variation. In addition, the cost of generators, Var compensators, and the peak power savings may be subject to uncertainty to some degree. Therefore, uncertainties due to insufficient information may generate an uncertain region of decisions. Consequently, the validity of the results from average values cannot represent the uncertainty level. To account for the uncertainties in information and goals related to multiple and usually conflicting objectives in RPP, the use of fuzzy set theory may play a significant role in decision-making.

A literature review of fuzzy set theory applied in power systems is presented in [49]. This survey introduces the basic principles of the theory, identifies problems suitable for fuzzy sets, and applies general approaches from fuzzy set theory in power system planning, operation, and control. Reference [25] presents an approach to handle the uncertainty of different goals, which is an important aspect related to MO optimization. The fuzzy sets may be assigned not only to objective functions, but also to constraints [50]-[52]. Also, the non-probabilistic uncertainty associated with the reactive power demand in constraints is discussed with fuzzy sets in [50]. Generally speaking, the satisfaction parameters (fuzzy sets) for objectives and constraints represent the degree of closeness to the optimum and the degree of enforcement of constraints, respectively [50], [51]. With the maximization of these satisfaction parameters, real power losses and Var cost are minimized, while enforcement of voltage security is maximized simultaneously, considering uncertainties [51].

Reference [53] applies the Bellman-Zadeh approach in decision making for MO optimization in a fuzzy environment in order to obtain harmonious solutions with equal or close levels of satisfying criteria. Thus, solutions with high levels of satisfying criteria that are reached by low levels of satisfying other 
TABLE I

Matrix of ObJectives, Models and Solution Algorithms for Reactive Power Planning

\begin{tabular}{|c|c|c|c|}
\hline Objective category & $\mathrm{OPF}(26)$ & SCOPF(6) & SCOPF-VS(4) \\
\hline Min Var cost & $\begin{array}{l}\text { [2] IPA } \\
\text { [3] Recursive MIP } \\
\text { [4] SLP, B\&B, PP } \\
\text { [5] SLP and GA }\end{array}$ & [29] SLP & $\begin{array}{l}\text { [35] NAG \& APEXIV, SM } \\
\text { [36] GAMS/DICOPT++, SM } \\
\text { [37] GAMS/DICOPT++, SM } \\
\text { [38] GAMS/DICOPT++, SM }\end{array}$ \\
\hline Min Var cost and real power losses & $\begin{array}{l}\text { [6] MINOS } \\
\text { [7] TS } \\
\text { [8] SLP } \\
\text { [9] EP } \\
\text { [10] HEP } \\
\text { [11] SLP, GA } \\
\text { [12] GA, SA } \\
\text { [13] TS } \\
\text { [50] Fuzzy logic } \\
\text { [51] Fuzzy logic } \\
\end{array}$ & $\begin{array}{l}\text { [30] SA } \\
\text { [31] BDM, PM/CM } \\
\text { [32] Recursive LP,PM }\end{array}$ & \\
\hline Min Var cost and generator fuel cost & $\begin{array}{l}\text { [14] SLP and GA } \\
\text { [15] CBA, MINOS } \\
\text { [16] EP, ES, GA }\end{array}$ & [33] BDM, CM & \\
\hline Min voltage deviation & [17] NLONN, AHP & & \\
\hline $\begin{array}{l}\text { Max the minimum singular } \\
\text { value of Jacobian matrix }\end{array}$ & [18] SA & & \\
\hline Max P-margin & [19] SQP & & \\
\hline $\begin{array}{c}\text { Min Var cost + real power } \\
\text { loss }+ \text { voltage deviation }\end{array}$ & $\begin{array}{l}\text { [20] TLSA } \\
{[21] \text { ESSA }}\end{array}$ & & \\
\hline $\begin{array}{l}\text { Min voltage deviation }+ \\
\text { generator Var deviation }+ \\
\text { real power losses }\end{array}$ & {$[22] \mathrm{GA}$} & & \\
\hline $\begin{array}{c}\text { Min Var cost + real power } \\
\text { losses + voltage deviation } \\
\text { + line flow deviation }\end{array}$ & & $\begin{array}{l}{[34] \varepsilon \text {-constraint method, }} \\
\text { SA }\end{array}$ & \\
\hline $\begin{array}{l}\text { Min real power losses+ Var source } \\
\text { cost }+ \text { voltage deviation } \\
\& \operatorname{Max} \\
\text { voltage stability margin (S-margin) }\end{array}$ & $\begin{array}{l}\text { [23] Goal-attainment, SA } \\
\text { [24] weighted- norm, SA } \\
\text { [25] Fuzzy logic, SA } \\
\text { [26] } \varepsilon \text {-constraint Method, SA }\end{array}$ & & \\
\hline $\begin{array}{l}\text { Min real power losses } \\
\quad+\text { Var source cost } \\
\text { \& Max PoC }\end{array}$ & [27] SPEA & & \\
\hline
\end{tabular}

Note:

AHP-Analytic Hierarchical Process

APEXIV - a software package

$\mathrm{B} \& \mathrm{~B}-$ Branch and Bound

BD-Bender Decomposition Method

CBA-Cost Benefit Analysis

CM-Corrective Mode

DICOPT++-MINLP solver in GAMS

EP-Evolutionary Programming

ES-Evolutionary Strategy

ESSA — hybrid Expert System Simulated Annealing

GA-Genetic Algorithm

GAMS - General Algebraic Modeling System, a modeling language

HEP-Hybrid Evolutionary Programming

IPA-Interior Point Algorithm

MIP_-Mixed Integer Programming

MINLP_-Mixed Integer NonLinear Programming

MINOS - a software package for large-scale linear and nonlinear optimization problems

NAG-Numerical Algorithm Group software package

NLONN-Nonlinear Optimization Neural Network

TS-Tabu Search

PM-Preventive Mode

PP_Parallel Processing

SA-Simulated Annealing Approach

SLP-Successive (Sequential) Linear Programming

SM-S Margin (MVA Stability Margin)

SPEA - Strength Pareto Evolutionary Algorithm

SQP_-Successive (Sequential) Quadratic Programming

TLSA-Two-Layer Simulated Annealing

criteria can be avoided. This approach has been initially applied to reactive power control and planning and may be explored further in the future.

Fuzzy set theory enables the integration of the effects of parameters' uncertainties into the analysis and offers a better compromised solution. In addition, it eliminates the need for many simulation runs. The fuzzy set method offers the decision maker with alternatives for selecting the Var location and Var size, which utilities can employ to make decisions regarding reactive power compensation in their systems.

\section{CONCLUSIONS}

This paper introduces the general background, objectives, constraints, and solution algorithms of Reactive Power Planning (RPP). The technologies in the literature of RPP are summarized in Table I. 
In summary, RPP is typically solved with an optimization model. The objective function may be the minimization of the cost, which includes the Var installation cost, power loss cost, and/or operation cost (generation fuel cost). For Var installation cost, the formulation of $C_{0}+C_{1} \cdot Q_{c}$ includes a fixed cost that may lead to the necessity of a binary variable to indicate whether the Var compensator will be installed or not. Hence, integer programming is needed in this case. With the ongoing deregulation in power industry, it is probably more reasonable to use Var cost + operation (generation fuel) cost as the objective than to use Var cost + power loss cost, since fuel cost minimization is typically applied in market operation and planning. In addition, the objective may consider some technical merits such as minimization of voltage deviation from a given schedule, maximization of voltage stability margin, or a MO model considering weighted sum of various costs and technical merits. It should be noted that some alternative approaches in RPP address RPP using sensitivity analysis based on various indices to avoid the complicated optimization formulation with the trade-off in accuracy. Not surprisingly, the sensitivity analysis may serve as a pre-screening to filter some less competitive solutions and then is combined with an optimization-based approach to find the best solution.

Under the present status of congested transmission system in the United States as well as many other power systems, the constraints should consider transmission security (or contingency). Also, voltage stability should be considered in RPP since a major goal of Var compensation is to improve voltage stability. Certainly, each of these two considerations requires much more computation effort in general. However, from a mathematical standpoint, the major modeling challenge lies in voltage stability constraints because the potential Var compensation will change the system voltage stability margin that in turn will impact the generation dispatch. Hence, the challenge lies in how to efficiently incorporate the expected new stability margin into the optimization model of Var location and amount without repeatedly running extra OPF or CPF to obtain the new voltage stability margin after Var compensation. As a comparison, the challenge of security constraints is mainly computational rather than in modeling, because each contingency case can be modeled in a way similar to the base case, although many contingency cases must be included.

As a typical optimization problem, RPP may be solved with classic optimization algorithms like LP, NLP, or MINLP. Due to the nonlinearity of power systems, LP loses accuracy due to linear assumptions. Consideration of nonlinear algorithms and integer variables will make the running time much longer and the algorithm possibly less robust. Newer algorithms based on intelligent searches such as SA, EA, and TS can address the integer variable very well. However, it is more heuristic than conventional optimization techniques and needs further investigation regarding performance under different systems. Another interesting aspect is to include fuzzy set theory to model the uncertainties in objective function as well constraints.

\section{ACKNOWLEDGMENT}

The authors would like to thank Oak Ridge National Laboratory for the financial support in part to accomplish this paper.

\section{REFERENCES}

[1] W. Zhang and L. M. Tolbert, "Survey of reactive power planning methods," in IEEE Power Engineering Society General Meeting, San Francisco, CA, Jun. 12-16, 2005, pp. 1580-1590.

[2] D. Pudjianto, S. Ahmed, and G. Strbac, "Allocation of VAR support using LP and NLP based optimal power flows," IEE Proc. Generation, Transmission, and Distribution, vol. 149, no. 4, pp. 377-383, 2002.

[3] K. Aoki, M. Fan, and A. Nishikori, "Optimal Var planning by approximation method for recursive mixed-integer linear programming," IEEE Trans. Power Syst., vol. 3, no. 4, pp. 1741-1747, Nov. 1988.

[4] J. R. S. Mantovani and A. V. Garcia, "A heuristic method for reactive power planning," IEEE Trans. Power Syst., vol. 11, no. 1, pp. 68-74, Feb. 1996.

[5] M. Delfanti, G. Granelli, P. Marannino, and M. Montagna, "Optimal capacitor placement using deterministic and genetic algorithms," IEEE Trans. Power Syst., vol. 15, no. 3, pp. 1041-1046, Aug. 2000.

[6] C. T. Hsu, Y. H. Yan, C. S. Chen, and S. L. Her, "Optimal reactive power planning for distribution systems with nonlinear loads," in Proc. IEEE Region 10 Int. Conf. Computer, Communication, Control and Power Engineering, Beijing, China, Oct. 19-21, 1993, vol. 5, pp. 330-333.

[7] W. Zhang, Y. Liu, and Y. Liu, "Optimal VAr planning in area power system," in Proc. Int. Conf. Power System Technology, Oct. 13-17, 2002, pp. 2072-2075.

[8] K. Iba, H. Suzuki, K. I. Suzuki, and K. Suzuki, "Practical reactive power allocation/operation planning using successive linear programming," IEEE Trans. Power Syst., vol. 3, no. 2, pp. 558-566, May 1988.

[9] L. L. Lai and J. T. Ma, "Application of evolutionary programming to reactive power planning-comparison with nonlinear programming approach," IEEE Trans. Power Syst., vol. 12, no. 1, pp. 198-206, Feb. 1997.

[10] V. Gopalakrishnan, P. Thirunavukkarasu, and R. Prasanna, "Reactive power planning using hybrid evolutionary programming method," in Proc. Power Systems Conf. \& Expo. 2004 IEEE PES, New York, Oct. 10-13, 2004, vol. 3, pp. 1319-1323.

[11] J. Urdaneta, J. F. Gomez, E. Sorrentino, L. Flores, and R. Diaz, “A hybrid genetic algorithm for optimal reactive power planning based upon successive linear programming," IEEE Trans. Power Syst., vol. 14, no. 4, pp. 1292-1298, Nov. 1999.

[12] A. H. H. Al-Mohammed and I. Elamin, "Capacitor placement in distribution systems using artificial intelligent techniques," in Proc. 2003 IEEE Power Tech Conf., Bologna, Italy, Jun. 23-26, 2003, vol. 4, p. 7.

[13] R. A. Gallego, A. J. Monticelli, and R. Romero, "Optimal capacitor placement in radial distribution networks," IEEE Trans. Power Syst., vol. 16, no. 4, pp. 630-637, Nov. 2001.

[14] K. Y. Lee, X. Bai, and Y. M. Park, "Optimization method for reactive power planning by using a modified simple genetic algorithm," IEEE Trans. Power Syst., vol. 10, no. 4, pp. 1843-1850, Nov. 1995.

[15] D. Chattopadhyay, K. Bhattacharya, and J. Parikh, "Optimal reactive power planning and its spot pricing: An integrated approach," IEEE Trans. Power Syst., vol. 10, no. 4, pp. 2014-2020, Nov. 1995.

[16] K. Y. Lee and F. F. Yang, "Optimal reactive power planning using evolutionary algorithms: A comparative study for evolutionary programming, evolutionary strategy, genetic algorithm, and linear programming," IEEE Trans. Power Syst., vol. 13, no. 1, pp. 101-108, Feb. 1998.

[17] J. Z. Zhu, C. S. Chang, W. Yan, and G. Y. Xu, "Reactive power optimization using an analytic hierarchical process and a nonlinear optimization neural network approach," IEE Proc. Generation, Transmission, and Distribution, vol. 145, no. 1, pp. 89-97, Jan. 1998.

[18] Y. L. Chen, "Weak bus oriented reactive power planning for system security," IEE Proc. Generation, Transmission and Distribution, vol. 143, no. 6, pp. 541-545, Nov. 1996.

[19] B. Kermanshahi, K. Takahashi, and Y. Zhou, "Optimal operation and allocation of reactive power resource considering static voltage stability," in Proc. 1998 Int. Conf. Power System Technology (POWERCON '98), Aug. 18-21, 1998, vol. 2, pp. 1473-1477.

[20] Y. L. Chen and Y. L. Ke, "Multi-objective Var planning for largescale power systems using projection-based two-layer simulated annealing algorithms," IEE Proc. Generation, Transmission and Distribution, vol. 151, no. 4, pp. 555-560, Jul. 11, 2004

[21] W. S. Jwo, C. W. Liu, C. C. Liu, and Y. T. Hsiao, "Hybrid expert system and simulated annealing approach to optimal reactive power planning," IEE Proc. Generation, Transmission and Distribution, vol. 142, no. 4, pp. 381-385, Jul. 1995.

[22] K. Iba, "Reactive power optimization by genetic algorithm," in Proc. Power Industry Computer Application Conf., May 4-7, 1993, pp. 195-201. 
[23] Y. L. Chen and C. C. Liu, "Multiobjective Var planning using the goal attainment method," IEE Proc. Generation, Transmission and Distribution, vol. 141, no. 3, pp. 227-232, May 1994.

[24] Y. L. Chen, "Weighted-norm approach for multiobjective Var planning," IEE Proc. Generation, Transmission and Distribution, vol. 145, no. 4, pp. 369-374, Jul. 1998.

[25] Y. L. Chen and C. C. Liu, "Interactive fuzzy satisfying method for optimal multi-objective Var planning in power systems," IEE Proc. Generation, Transmission and Distribution, vol. 141, no. 6, pp. 554-560, Nov. 1994.

[26] Y. L. Chen and C. C. Liu, "Optimal multi-objective Var planning using an interactive satisfying method," IEEE Trans. Power Syst., vol. 10, no. 2, pp. 664-670, May 1995.

[27] R. Ramos, J. Vallejos, and B. Barán, "Multi-objective reactive power compensation with voltage security," in Proc. 2004 IEEE/PES Transmission and Distribution Conf. and Expo.: Latin America, Brazil, Nov. 2004, pp. 302-307.

[28] R. C. Dageneff, W. Neugebauer, and C. Saylor, "Security constrained optimization: An added dimension in utility systems optimal power flow technology," IEEE Comput. Appl. Power, pp. 26-30, Oct. 1988.

[29] W. R. Thomas, A. M. Dixon, D. T. Y. Cheng, R. M. Dunnett, G. Schaff, and J. D. Thorp, "Optimal reactive planning with security constraints," in Proc. IEEE Power Industry Computer Application Conf., May 7-12, 1995, pp. 79-84.

[30] Y. T. Hsiao, C. C. Liu, H. D. Chiang, and Y. L. Chen, "A new approach for optimal Var sources planning in large scale electric power systems," IEEE Trans. Power Syst., vol. 8, no. 3, pp. 988-996, Aug. 1993.

[31] T. Gomez, I. J. Perez-Arriaga, J. Lumbreras, and V. M. Parra, "A security-constrained decomposition approach to optimal reactive power planning," IEEE Trans. Power Syst., vol. 6, no. 3, pp. 1069-1076, Aug. 1991.

[32] B. Cova et al., "Contingency constrained optimal reactive power flow procedures for voltage control in planning and operation," IEEE Trans. Power Syst., vol. 10, no. 2, pp. 602-608, May 1995.

[33] S. Granville, M. V. P. Pereira, and A. Monticelli, "An integrated methodology for Var sources planning," IEEE Trans. Power Syst., vol. 3, no. 2, pp. 549-557, May 1988 .

[34] Y. T. Hsiao, H. D. Chiang, C. C. Liu, and Y. L. Chen, "A computer package for optimal multi-objective VAR planning in large scale power systems," IEEE Trans. Power Syst., vol. 9, no. 2, pp. 668-676, May 1994.

[35] O. O. Obadina and G. J. Berg, "Var planning for power system security," IEEE Trans. Power Syst., vol. 4, no. 2, pp. 677-686, May 1989.

[36] B. B. Chakrabarti, D. Chattopadhyay, and C. Krumble, "Voltage stability constrained Var planning-a case study for New Zealand," in Proc. LESCOPE '01 Large Engineering Systems Conf. Power Engineering, Jul. 11-13, 2001, pp. 86-91.

[37] D. Chattopadhyay and B. B. Chakrabarti, "Reactive power planning incorporating voltage stability," Int. J. Elect. Power and Energy Syst., vol. 24, no. 3, pp. 185-200, 2002.

[38] D. Chattopadhyay and B. B. Chakrabarti, "Voltage stability constrained Var planning: Model simplification using statistical approximation," Int. J. Elect. Power and Energy Syst., vol. 23, no. 5, pp. 349-358, 2001

[39] O. Alsac and B. Stott, "Optimal load flow with steady-state security," IEEE Trans. Power App. Syst., vol. PAS-93, pp. 745-751, May/Jun. 1974.

[40] W. C. Merritt, C. H. Saylor, R. C. Burchett, and H. H. Happ, "Security constrained optimization-a case study," IEEE Trans. Power Syst., vol. 3, no. 3, pp. 970-977, Aug. 1988.

[41] W. Qiu, A. J. Flueck, and F. Tu, "A new parallel algorithm for security constrained optimal power flow with a nonlinear interior point method," in IEEE Power Engineering Society General Meeting, San Francisco, CA, Jun. 12-16, 2005, pp. 2422-2428.

[42] L. G. Dias and M. E. El-Hawary, "Security-constrained OPF: Influence of fixed tap transformer fed loads," IEEE Trans. Power Syst., vol. 6, no. 4, pp. 1366-1372, Nov. 1991.

[43] V. C. Ramesh and X. Li, "A fuzzy multiobjective approach to contingency constrained OPF," IEEE Trans. Power Syst., vol. 12, no. 3, pp. 1348-1354, Aug. 1997.

[44] V. Ajjarapu and C. Christy, "The continuation power flow: A tool for steady state voltage stability analysis," IEEE Trans. Power Syst., vol. 7, no. 1, pp. 416-423, Feb. 1992.

[45] C. A. Caîzares, "Calculating optimal system parameters to maximize the distance to saddle-node bifurcations," IEEE Trans. Circuits Syst. I, vol. 45, no. 3, pp. 225-237, Mar. 1998.

[46] C. A. Caĩzares, "Applications of optimization to voltage collapse analysis," in Panel Session: Optimization Techniques in Voltage Collapse Analysis, IEEE-PES Summer Meeting, San Diego, CA, Jul. 1998.
[47] V. Ajjarapu, P. L. Lau, and S. Battula, "An optimal reactive power planning strategy against voltage collapse," IEEE Trans. Power Syst., vol. 9, no. 2, pp. 906-917, May 1994.

[48] T. Van Cutsem, "A method to compute reactive power margins with respect to voltage collapse," IEEE Trans. Power Syst., vol. 6, no. 1, pp. 145-156, Feb. 1991

[49] J. A. Momoh, X. W. Ma, and K. Tomsovic, "Overview and literature survey of fuzzy set theory in power systems," IEEE Trans. Power Syst., vol. 10, no. 3, pp. 1676-1690, Aug. 1995.

[50] K. H. Abdul-Rahman and S. M. Shahidehpour, "Application of fuzzy sets to optimal reactive power planning with security constraints," IEEE Trans. Power System, vol. 9, no. 2, pp. 589-597, May 1994.

[51] B. Venkatesh, G. Sadasivam, and M. A. Khan, "An efficient multi-objective fuzzy logic based successive LP method for optimal reactive power planning," Elect. Power Syst. Res., vol. 59, no. 2, pp. 89-102, Sept. 28, 2001.

[52] P. Y. Ekel and F. H. S. Neto, "Algorithms of discrete optimization and their application to problems with fuzzy coefficients," Info. Sci., vol. 176, pp. 2846-2868, 2006.

[53] P. Y. Ekel, C. A. P. S. Martins, J. G. Pereira, Jr., R. M. Palhares, and L. N. Canha, "Fuzzy set based multiobjective allocation of resources and its applications," Int. J. Comput. Math. Applicat., vol. 52, no. 1-2, pp. 197-210, 2006.

[54] F. F. Wu, G. Gross, J. F. Luini, and P. M. Look, "A two-stage approach to solving large-scale optimal power flows," in Proc. IEEE Power Industry Computer Applications Conf. 1979 (PICA-79), May 15-18, 1979 , pp. $126-136$.

[55] R. V. V. Vidal, Applied Simulated Annealing. New York: SpringerVerlag, 1993

[56] D. T. Pham and D. Karaboga, Genetic Algorithms, Tabu Search, Simulated Annealing and Neural Networks. New York: Springer, 2000.

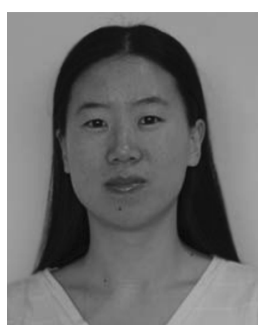

Wenjuan Zhang (S'03) received the B.S.E.E. degree from Hebei University of Technology, Hebei, China, in 1999, and the M.S.E.E. degree from Huazhong University of Science and Technology, China, in 2003. She received the Ph.D. degree in electrical engineering at The University of Tennessee, Knoxville, in 2007.

She is presently working at the California ISO, Folsom. Her research interests include reactive power compensation and planning, voltage stability, and optimization.

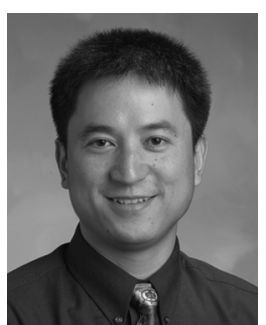

Fangxing (Fran) Li (M'01-SM'05) received the $\mathrm{Ph} . \mathrm{D}$. degree from Virginia Tech, Blacksburg, in 2001

He has been an Assistant Professor in the ECE Department, The University of Tennessee (UT), Knoxville, since August 2005. He was previously with ABB Inc., Raleigh, NC, as a Senior and then Principal Engineer prior to joining UT. His current interests include reactive power, distributed generation, energy markets, reliability, computational methods, and power electronics.

Dr. $\mathrm{Li}$ is the recipient of the 2006 Eta Kappa Nu Outstanding Teacher Award at UT. He is a Registered Professional Engineer in the state of North Carolina.

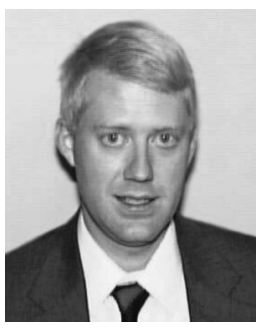

Leon M. Tolbert (SM'99) received the B.S., M.S., and Ph.D. degrees from Georgia Tech, Atlanta, in 1989, 1991, and 1999, respectively.

He joined the Engineering Division of Oak Ridge National Laboratory (ORNL), Oak Ridge, TN, in 1991. He was appointed as an Assistant Professor in the ECE Department at The University of Tennessee (UT), Knoxville, in 1999. He is presently an Associate Professor at UT and a Research Engineer at ORNL.

Dr. Tolbert was Chairman of the Education Activities Committee of the IEEE Power Electronics Society from 2003 to 2007. He is a Registered Professional Engineer in the state of Tennessee. 